\title{
EFFECT OF RANDOM GEOMETRIC UNCERTAINTY ON THE COMPUTATIONAL DESIGN OF A 3-D FLEXIBLE WING
}

\author{
C. R. Gumbert \\ NASA Langley Research Center, Hampton, VA 23681-2199 \\ P. A. Newman \\ NASA Langley Research Center, Hampton, VA 23681-2199 \\ G. J.-W. Hou ${ }^{\ddagger}$ \\ Old Dominion University, Norfolk, VA 23529-0247
}

\begin{abstract}
The effect of geometric uncertainty due to statistically independent, random, normally distributed shape parameters is demonstrated in the computational design of a 3-D flexible wing. A first-order second-moment statistical approximation method is used to propagate the assumed input uncertainty through coupled Euler CFD aerodynamic / finite element structural codes for both analys is and sensitivity analysis. First-order sensitivity derivatives obtained by automatic differentiation are used in the input uncertainty propagation. These propagated uncertainties are then used to perform a robust design of a simple 3-D flexible wing at supercritical flow conditions. The effect of the random input uncertainties is shown by comparison with conventional deterministic design results. Sample results are shown for wing planform, airfoil section, and structural sizing variables.
\end{abstract}

\section{Introduction}

"A robust design problem is one in which a design is sought that is relatively insensitive to uncertain quantities" [1]; that is, the objective function does not change much and the constraints are not likely to be violated. Robust design problems are prevalent in disciplines such as structures [2] and mechanisms [3], but, due to lack of study to quantify uncertainty in aerodynamics and computational expense, have not been widely applied for aerodynamic design. This paper represents an attempt to study robust design of a flexible wing that is built upon an established multidisciplinary design optimization procedure.

Several recent papers [4-8] have addressed the issues of and obtained sample results using CFD for aerodynamic optimizations subject to uncertainties in the input or design variables; we shall call these robust

* Research Scientist, Multidisciplinary Optimization Branch, M/S 159, c.r.gumbert@1arc.nasa.gov

$\dagger$ Senior Research Scientist, Multidisciplinary Optimization Branch, M/S 159, p.a.newman@larc.nasa.gov

¥ Professor, Department of Mechanical Engineering, AIAA member, ghou@lions.odu.edu

Copyright (C) 2002 by the American Institute of Aeronautics and Astronautics, Inc. No copyright is asserted in the United States under Title 17, U.S. Code. The U.S. Government has a royalty-free license to exercise all rights under the copyright claimed herein for Governmental purposes. All other rights are reserved by the copyright owner. optimizations in contrast to conventional or deterministic optimizations. In all of these robust optimization demonstrations, three steps are involved. First, the input uncertainties are quantified. Second, the input uncertainties are propagated through the CFD code to obtain uncertainties for the output functions. Third, the output functions with uncertainties are used in the optimization objective and constraint functions to perform a robust design. Implementation algorithms and details used to accomplish these three steps differ in the cited papers, but in all cases the input uncertainty is shown to influence the design. These input uncertainties represent error sources that are "external" to the CFD code. Several other recent papers [9-14] have discussed issues and results related to uncertainty analysis for CFD codes and applications but have not included optimization or design.

Computational simulation uncertainties also arise from physical, mathematical, and numerical modeling approximations (see, for example, [11-17]); these are called "internal" model error and uncertainty sources. Internal sources are not considered herein but must be included in assessing the total uncertainty in any computational simulation of physical phenomena. The recent Journal of Fluids Engineering special section on "Quantifying Uncertainty in CFD" [15] contains eight invited papers addressing both numerical accuracy and physical uncertainty issues. In the present work, the discrete code results are considered 
to be deterministically certain in order to show the influence of statistically random input geometry, such as may be due to manufacturing variations, on a gradient-based performance design optimization. As pointed out in [5], [7], and [8], these probabilistic-based performance designs differ from the probabilistic-based reliability designs that are usually considered in the structures disciplines. For the former problem, one is more interested in the probable or frequent events that occur around the mean values, whereas for the latter problem, one is most interested in the infrequently occurring catastrophic events. An example of multidisciplinary performance optimization subject to uncertainty involving linear aerodynamics can be found in [18]; simultaneous consideration of both types of probabilistic-based design problems is also discussed there.

The present work considers the simultaneous aerodynamic-structural performance optimization of a simple 3-D flexible wing subject to statistical uncertainty in geometric input for wing planform, airfoil section, and structural thickness design variables. The approach presented in [5] and [8] is extended to multidimensional supercritical flow about a flexible wing that is modeled by high fidelity multidisciplinary computational simulations. A first-order secondmoment (FOSM) statistical approximation method is used to propagate the assumed input uncertainty through coupled Euler CFD aerodynamic/finite element structural codes for both system analysis and sensitivity analysis. First-order sensitivity derivatives (SD) obtained by automatic differentiation are used in the FOSM input uncertainty propagation. These propagated uncertainties are then used to perform a robust design of a simple $3-\mathrm{D}$ flexible wing. The effect of the random input uncertainties is shown by comparing it with conventional deterministic design results. This present implementation of the statistical approach is very easy to retrofit into gradient-based design codes that already utilize analytical or semianalytical sensitivity derivatives for optimization.

\section{Integrated Statistical Approach}

The integrated statistical approach, as demonstrated for a CFD code in [5] and [8] for quasi 1-D subsonic Euler flow, is presently implemented for supercritical flow about a 3-D flexible wing. This approach follows the integrated strategy of [3] that was demonstrated on a linkage mechanism design. That strategy for mitigating the effect of uncertainty includes (a) uncertainty quantification, (b) uncertainty propagation, and (c) robust design. Herein, the details differ somewhat from [3], [5], and [8]; the major difference is in the process of obtaining the required second-order sensitivity derivatives. In this present implementation, finite differencing of the "robust" objective and active constraint functions is controlled by the optimizer code, whereas in the quasi 1-D application $[5,8]$, these second derivative pieces were constructed using both hand- and automatically differentiated code as detailed in [19] and provided to the optimizer code.

\section{Uncertainty Quantification}

Uncertainty quantification is not an easy task (for example, see [6]); though it is an extremely important one that must be included for any realistic design. The authors have neither the manufacturing data nor the engineering experience to address it here. The uncertainty quantification issue was simulated here by assuming that the uncertainty in geometry was characterized by statistically independent, random, normally distributed shape parameters such as those due to manufacturing process variations. Our assumption simplifies the resulting algebra and equations to be coded, but it serves to illustrate the influence of input geometry on a 3-D flexible wing design.

\section{Uncertainty Propagation}

Uncertainty propagation was accomplished herein using only the FOSM method, whereas in [5] and [8] both first- and second-order approximations were demonstrated and compared to Monte Carlo simulations. This propagation was done for both CFD Euler and finite element method (FEM) structures discipline codes using the sensitivity analysis codes from our Simultaneous Aerodynamic and Structural Design Optimization (SASDO) studies [20]. Firstorder SDs obtained by automatic differentiation have been used as previously demonstrated for a CFD code in [5] and [8] and an FEM code in [21]. A brief outline of the FOSM follows; for more details see [5] and [8].

The first step in the FOSM analyses is to approximate the system output solutions of interest in Taylor series form. These approximations are formed to estimate the output value for small deviations of the input. Given input random variables $\mathbf{b}=\left\{b_{1}, \ldots, b_{n}\right\}$ with means $\overline{\mathbf{b}}=\left\{\overline{\mathrm{b}}_{1}, \ldots, \overline{\mathrm{b}}_{\mathrm{n}}\right\} \quad$ and standard deviations $\sigma_{\mathrm{b}}=\left\{\sigma_{\mathrm{b}_{1}}, \ldots, \sigma_{\mathrm{bn}}\right\}$, and system output function $\mathrm{F}$, the first-order Taylor series approximations are

$$
\mathrm{F}(\mathbf{b})=\mathrm{F}(\overline{\mathbf{b}})+\sum_{i=1}^{n} \frac{\partial \mathrm{F}}{\partial \mathrm{b}_{\mathrm{i}}}\left(\mathrm{b}_{\mathrm{i}}-\overline{\mathrm{b}}_{\mathrm{i}}\right)
$$

One then obtains expected values for the mean (first moment) and variance (second moment) of the output function, F, which depend on the SD and input variances $\sigma_{\mathbf{b}}$. The mean of the output function $\overline{\mathrm{F}}$, and standard deviation $\sigma_{\mathrm{F}}$, are approximated as 


$$
\begin{aligned}
& \bar{F}=F(\overline{\mathbf{b}}) \\
& \sigma_{\mathrm{F}}{ }^{2}=\sum_{\mathrm{i}=1}^{\mathrm{n}}\left(\frac{\partial \mathrm{F}}{\partial \mathrm{b}_{\mathrm{i}}} \sigma_{\mathrm{b}_{\mathrm{i}}}\right)^{2}
\end{aligned}
$$

where the SD, $\partial \mathrm{F} / \partial \mathrm{b}_{\mathrm{i}}$, are evaluated at the mean values $\overline{\mathbf{b}}$. These SD are obtained using the automatic differentiation tool ADIFOR [22, 23]. Note that, although it is not shown explicitly, the function F may also depend on other parameters for which there is no uncertainty or for which that uncertainty is too small to be significant. Such variables would have no contribution to the standard deviation, $\sigma_{F}$. They are not explicitly included in any further discussion.

\section{Robust Design}

Conventional optimization for an objective function $\Psi$, which is a function of some system output $F$, state variables $\mathbf{Q}$, and input variables $\mathbf{b}$, is expressed in Eqs. (3)

$$
\begin{aligned}
& \min \Psi=\Psi(F ; \mathbf{Q}, \mathbf{b}) \\
& \text { subject to } \\
& \mathbf{g}(\mathrm{F} ; \mathbf{Q}, \mathbf{b}) \leq 0
\end{aligned}
$$

The CFD state and structural equilibrium equation residuals, $\mathbf{R}(\mathbf{Q}, \mathbf{b})=\mathbf{0}$, determine $\mathbf{Q}$ given $\mathbf{b}$. The system constraints $\mathbf{g}$ are represented as inequality constraints. The input variables $\mathbf{b}$ are precisely known for conventional optimization, and all functions of $\mathbf{b}$ are therefore deterministic.

For robust design, the conventional optimization must be treated in a probabilistic manner. Given uncertainty in the input variables $\mathbf{b}$, all of the functions in Eqs. (3) become uncertain. The design variables are now the mean values, $\overline{\mathbf{b}}=\left\{\overline{\mathrm{b}}_{1}, \ldots, \overline{\mathrm{b}}_{\mathrm{n}}\right\}$, with $\overline{\mathbf{b}}$ assumed statistically independent and normally distributed with standard deviations $\sigma_{\mathbf{b}}$. The CFD state and structural equilibrium equation residuals $\mathbf{R}$ are deemed to be satisfied at the mean values $\overline{\mathbf{Q}}$ and $\overline{\mathbf{b}}$ such that $\mathbf{R}(\overline{\mathbf{Q}}, \overline{\mathbf{b}})=0$. The objective function is cast in terms of expected values and becomes a function of $\overline{\mathrm{F}}$ and $\sigma_{\mathbf{F}}$. The system constraints are cast into a probabilistic statement: the probability that the constraints are satisfied is greater than or equal to a desired or specified target probability, that is $\mathrm{P}(\mathbf{g} \leq 0)>\mathrm{P}_{t}$. This probability statement is transformed [3] to a constraint involving mean values and standard deviations under the assumption that the variables involved are normally distributed. The robust optimization can be expressed as

$$
\begin{aligned}
& \min \Psi=\Psi\left(\overline{\mathrm{F}}, \boldsymbol{\sigma}_{\mathrm{F}} ; \overline{\mathbf{Q}}, \overline{\mathbf{b}}\right) \\
& \text { subject to } \\
& \mathbf{g}(\overline{\mathrm{F}} ; \overline{\mathbf{Q}}, \overline{\mathbf{b}})+\mathrm{k} \boldsymbol{\sigma}_{\mathrm{g}} \leq 0
\end{aligned}
$$

where $\mathrm{k}$ is the number of standard deviations $\sigma_{\mathbf{g}}$ that the constraint $\mathbf{g}$ must be displaced to achieve the desired or specified target probability $\mathrm{P}_{\mathrm{t}}$. For the FOSM approximation, the standard deviations $\sigma_{F}$ and $\sigma_{\mathbf{g}}$ are of the form given in Eqs. (2) involving firstorder SDs. Therefore, a gradient-based optimization will then require second-order SDs to compute the objective and constraint gradients. Herein, the probabilistic objective and constraint functions of Eqs. (4) are evaluated using function analysis and first-order SD values from sensitivity analysis codes. These augmented functions are then finite differenced to obtain the gradients as required by the optimization code.

\section{Application to 3-D Flexible Wing}

A simple 3-D flexible wing geometry is chosen to illustrate the influence that statistically random geometric shape and sizing parameters have on a gradient-based performance optimization for supercritical flow conditions. This application is multidisciplinary; it considers both aerodynamics and structures. The state vector $\mathbf{Q}$ considered above can be decomposed into a separate flow-field vector $\mathbf{Q}$ and a structural displacement vector $\mathbf{u}, \mathbf{Q}=\{\mathbf{Q}, \mathbf{u}\}$. The set of design variables $\mathbf{b}$ can also be separated into two sets: those describing the structural element sizes $\mathbf{b}_{\text {size }}$ and those describing the wing geometry $\mathbf{b}_{\text {geom }}$ such that $\mathbf{b}=\left\{\mathbf{b}_{\text {geom }}, \mathbf{b}_{\text {size }}\right\}$. The state equations can be separated as $\mathbf{R}=\left\{\mathbf{R}_{\mathrm{i}, \mathrm{j}, \mathrm{k}, 1}, \mathbf{R}_{\mathrm{m}}\right\}$ where the solution of the high fidelity Euler CFD model is represented by

$$
\mathbf{R}_{\mathrm{i}, \mathrm{j}, \mathrm{k}, \mathrm{l}}\left(\mathbf{Q}, \mathbf{X}_{\text {def }}\left(\mathbf{b}_{\text {geom }}, \mathbf{u}\right)\right)=0
$$

with $i, j, k$, and 1 representing loop indices over the volume mesh points and the mass, momentum, and energy equations and the solution of the linear FEM structural model is represented by

$$
\mathbf{R}_{\mathrm{m}}=\mathbf{K}\left(\mathbf{b}_{\text {size }}, \mathbf{X}_{\mathrm{jig}}\left(\mathbf{b}_{\text {geom }}\right)\right) \mathbf{u}-\mathbf{f}(\mathbf{Q})=0
$$

with $m$ representing the loop index over the FEM mesh points, $\mathbf{X}_{\mathrm{jig}}$. These equations are coupled in that the aerodynamic load $\mathbf{f}(\mathbf{Q})$ influences the displacement $\mathbf{u}$ of the structure and this displacement of the aerodynamic shape $\mathbf{X}_{\text {def }}$ changes the load. Sensitivity analysis essentially involves solving for the derivative of Eqs. (5) and (6) with respect to each of the design variables $\mathbf{b}_{\mathbf{i}}$ for $\partial \mathbf{Q} / \partial \mathbf{b}$ and $\partial \mathbf{u} / \partial \mathbf{b}$. Then the derivative equations are coupled by $\partial \mathbf{f} / \partial \mathbf{b}$ and $\partial \mathbf{u} / \partial \mathbf{b}$.

\section{Problem Description}

Two sample problems are considered: a two-designvariable (2DV) case for which conventional or deterministic optimization results have been previously obtained [24], and a four-design-variable (4DV) case. The trapezoidal-planform, semispan wing and input design variables are shown in Fig. 1. In the 
2DV examples, the wing sections are held fixed and vary linearly from an NACA 0012 at the root to an NACA 0008 at the tip, which is then rounded. The size of the structural elements is also held fixed in the 2DV examples. The two planform design variables are the tip setback $x_{t}$ and the tip chord $c_{t}$. Although these variables are not typically subject to significant variance, they are ascribed some uncertainty here to illustrate the technique and process of propagating input uncertainty through the coupled aerodynamic/structural analysis and optimizing the design in the presence of the uncertainty in the output functions.

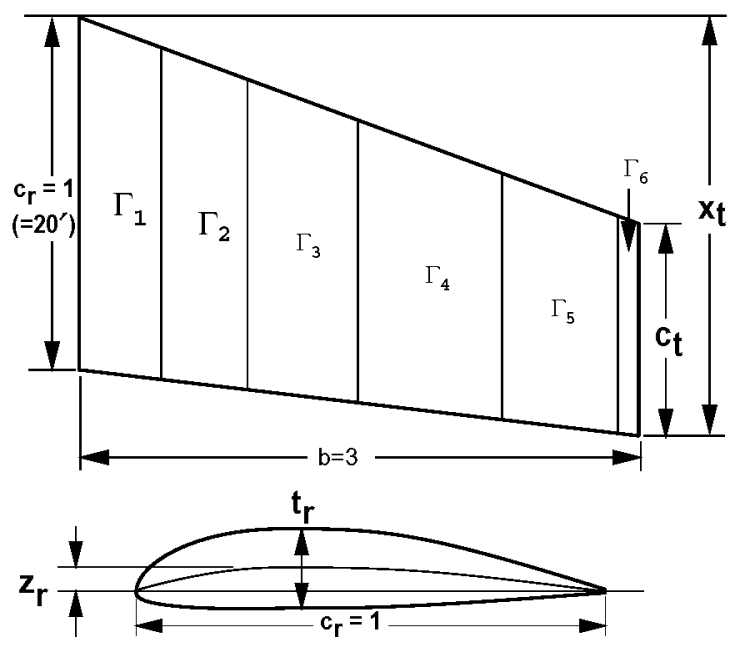

Figure 1. Wing geometry and sizing parameterization.

The relative sizes of the skin thickness, the web thicknesses, and the truss cross section areas are fixed within each structural zone depicted in Fig. 1. A scaling factor $\Gamma_{\mathrm{n}}$ is assigned to change the thickness and area of all structural elements in zone $\mathrm{n}$. In the $4 \mathrm{DV}$ case, two of these sizing factors are considered as design variables. The other two design variables considered in the 4DV case were determined by examining both sensitivity derivatives and (reasonable) expected variances. As can be seen from Eq. (2), the size of an output function variance depends on the size of the input variances weighted by the sensitivity derivative of the output function with respect to that input variable. For input variances of $0.1 \%$, the larger SDs with respect to root airfoil thickness $t_{r}$ and camber $\mathrm{Z}_{\mathrm{r}}$ appeared to provide a more realistic representation of practical uncertainty than the variables used in the $2 \mathrm{DV}$ cases.

The objective function to be minimized is the negative of the square of the lift-to-drag ratio, $-(\mathrm{L} / \mathrm{D})^{2}$, for $M_{\infty}=0.8, \alpha=1^{\circ}$, representative of cruise conditions for a transport aircraft. Both coupled solution- dependent and geometric constraints are imposed. The solution-dependent constraints are

- lower limit on the difference between the total lift and the structural weight, $L-W$, where $\mathrm{L}=\mathrm{C}_{\mathrm{L}} \mathrm{q}_{\infty} \mathrm{S}$

- upper limit on compliance, the work done by the aerodynamic loads to deflect the structure, $\mathrm{V}=\int \mathrm{f} \mathrm{pu} \cdot \hat{\mathbf{n}} \mathrm{ds}$

- upper limit on pitching moment, $\mathrm{C}_{\mathrm{m}}$, in lieu of a trim constraint

The purely geometric constraints are

- minimum leading edge radius, in lieu of a manufacturing requirement

- side constraints (bounds) on the active design variables

State variables for aerodynamics and structures are determined from Eqs. (5) and (6), the fluid-flow conservation laws (Euler equations) and the structural equilibrium conditions (for FEM), respectively. The conventional or deterministic optimization for the 2DV case follows Eqs. (3) and is reported in [24]. Details regarding procedures, equations, codes, and background references are given in that paper and will not be repeated here.

\section{Robust Design Process}

Robust design for both cases follows the integrated statistical approach as outlined above, i.e. Eqs. (1), (2), and (4), according to [5] and [8]. The objective and constraint functions listed above are approximated by Eqs. (1) with their mean and variances given by Eqs. (2). First-order SDs, as obtained from ADIFORprocessed analysis code, are used in Eqs. (2) to propagate the input uncertainty to the output of this coupled multidisciplinary system. Figure 2 depicts a

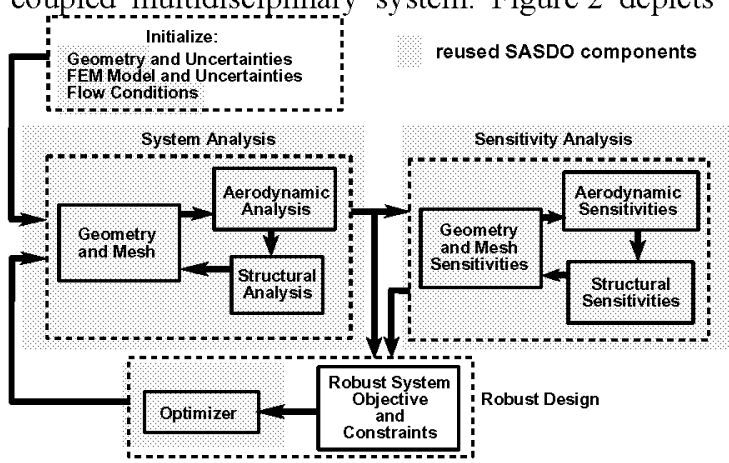

Figure 2. Schematic of robust optimization process.

schematic of this integrated procedure and data flow for robust design optimization according to Eqs. (4). The shaded portions of the schematic depicted in Fig. 2 represent code blocks unchanged from the original deterministic gradient-based design code [20, 24]. 
In the simple CFD example considered in [5] and [8], the objective function was for matching a target, i.e., to minimize the square of the difference between an output and a predetermined target value. In the construction of the objective function obtained by taking expected values, the FOSM approximation of the variance survives because of the squaring operation. Thus, first-order SDs appear in the robust objective function. In the present example, the objective function is taken as $-(\mathrm{L} / \mathrm{D})^{2}$ and again the FOSM approximation to the variance appears in the robust version. Then when the FOSM approximation of the standard deviation is used in the constraint, the robust optimization problem is given as

$$
\begin{aligned}
& \min \Psi=-(\overline{\mathrm{L}} / \overline{\mathrm{D}})^{2}-\sum_{\mathrm{i}=1}^{\mathrm{n}}\left(\frac{\partial(\mathrm{L} / \mathrm{D})}{\partial \mathrm{b}_{\mathrm{i}}} \boldsymbol{\sigma}_{\mathrm{b}_{\mathrm{i}}}\right)^{2} \\
& \text { subject to } \\
& \mathbf{g}=\overline{\mathbf{g}}+\mathrm{k} \sqrt{\sum_{\mathrm{i}=1}^{\mathrm{n}}\left(\frac{\partial \mathbf{g}}{\partial \mathrm{b}_{\mathrm{i}}} \boldsymbol{\sigma}_{\mathrm{b}_{\mathrm{i}}}\right)^{2}} \leq 0
\end{aligned}
$$

We note here that the robust optimization problem reduces to the conventional or deterministic problem when the standard deviation of the inputs $\sigma_{\mathrm{b}}$ is zero. Specific computational tools used to perform the tasks depicted by the solid boxes in Fig. 2 are identified in the next section.

\section{Computational Tools}

Major computations in this robust optimization procedure are performed using a collection of existing codes. These codes are executed by a separate driver code and scripts. Each code runs independently, some simultaneously on separate processors, and the required data transfers between them, also directed by the driver, are accomplished via data files.

The aerodynamic flow analysis code used for this study is a version of the CFL3D code [25] used in the Euler mode. The gradient version of this code, which was used for aerodynamic sensitivity analysis, was generated by an unconventional application [26] of the automatic differentiation code $\operatorname{ADIFOR}[22,23]$ to produce a relatively efficient, direct mode, gradient analysis code [27].

The surface geometry was generated based on a code utilizing the Rapid Aircraft Parameterization Input Design (RAPD) technique developed by Smith et al [28]. This code was also preprocessed with ADIFOR to generate a code capable of producing SD as well.

The CFD volume mesh needed by the flow analysis code was generated using a version of the CSCMDO [29] grid generation code. The associated grid SDs needed by the flow sensitivity analysis were generated with an automatically differentiated version of
CSCMDO [30]. The 45,000 grid point baseline volume mesh required by CSCMDO and used in the present flexible wing examples was obtained with the Gridgen $^{\mathbf{T M}}$ code. The wing surface portion of the mesh is shown in Fig. 3. This mesh is admittedly quite coarse by current CFD analysis standards.

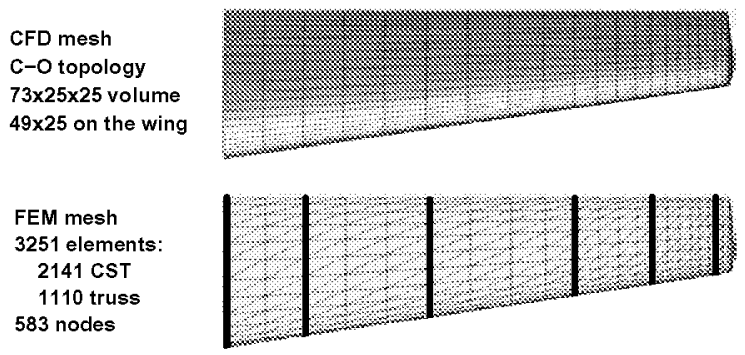

Figure 3. CFD and FEM computational meshes.

The structural analysis code [31] used to compute the deflection of the elastic wing was a generic finite element code. The flexible structure for the wing shown in Fig. 3 was discretized by 583 nodes; there were 2,141 constant-strain triangle (CST) elements and 1,110 truss elements. Zone boundaries for the design variables controlling element size are also shown in Fig. 3. Because the elastic deformation was assumed to be small, linear elasticity was deemed to be appropriate. The structural sensitivity equations were derived based on the direct differentiation method. The sensitivity of the aerodynamic forces appears as a term on the right-hand side (RHS) of the deflection sensitivity equations. The derivative of the stiffness matrix in these sensitivity equations was also generated [32] by using the ADIFOR [22, 23] technique. The coefficient matrix of the structural sensitivity equations was identical to that of the structural equations. Consequently, these structural sensitivity equations were solved efficiently by backward substitution with different RHSs for each sensitivity.

At the wing surface, i.e., the interface where aerodynamic load and structural deflection information is transferred, surface nodes of the FEM structural model were assumed to be a subset of the CFD aerodynamic surface mesh points for this application (see Fig. 3). This lack of generality allowed for simplifications in the data transfers and, although an important issue, it was not deemed crucial for these initial 3-D robust optimization demonstrations.

The code for the box labeled Robust System Objective and Constraints was simply the programming of Eqs. (1), (2) and (4) as previously discussed. These probabilistic objective and constraints were then fed to the optimizer [33]. The Sequential Quadratic Programming (SQP) procedure was used and the 
objective and constraint gradients were calculated by the optimizer using finite differences.

\section{Sample Results \& Discussion}

\section{Two-Design-Variable Cases}

The 2DV optimization problem was solved using several values for the input uncertainties associated with the design variables. Those results are compared to conventional or deterministic design results in Figs. 4. Figure 4(a) shows the level sets (contours) of the deterministic objective function shaded according to the key and drawn as thin solid black lines. In addition, the deterministic payload constraint function is shown as a yellow (light) solid shaded region to indicate where it is violated and as contour lines to indicate where it is satisfied. The square symbol is the deterministic optimization result. For the deterministic design, the payload constraint is active, but the compliance and pitching moment constraints are not; in fact, with the initial design point of $(1,1)$ they were never active during the deterministic optimization process. This deterministic result was the initial design point from which all the robust optimization problems were started. For all the robust design problems, it was assumed that the same value $\sigma$ could be used for each of the input $\sigma_{\mathrm{i}}$.

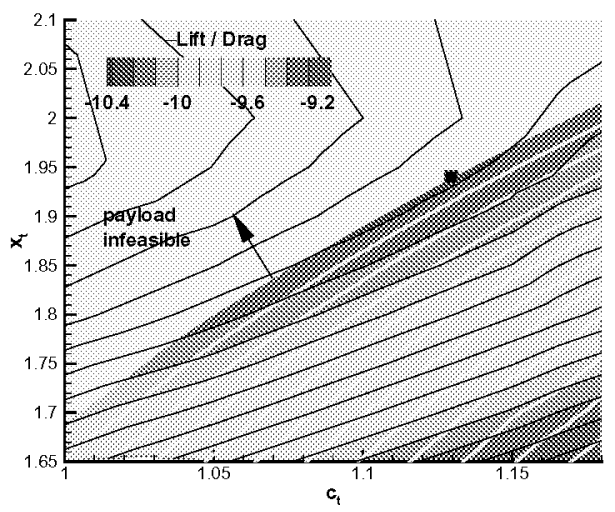

Figure 4(a). 2DV level sets of deterministic objective and active constraint functions with conventional optimization result.

Figure 4(b) shows robust optimization results overlaid on the deterministic results. The robust designs with $\sigma=0.01$ and $\sigma=0.02$ have been pushed away from the payload constraint boundary during the optimization process. The level sets for the deterministic objective function and payload constraint are nearly parallel over a significant region so that there is a large allowable variation in the design variables to solve the design problem to the specified tolerance. This can also be recognized by the solution for a second deterministic optimization problem shown as the circle for which the starting point was the solution of the problem with $\sigma=0.02$.

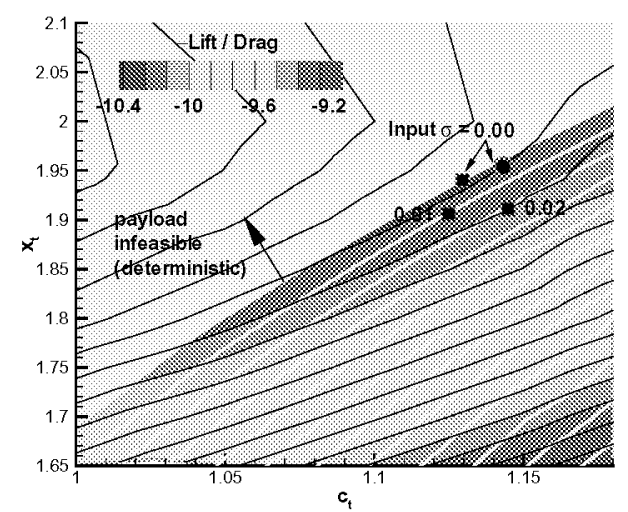

Figure 4(b). Probabilistic $\sigma=0.01 \& 0.02$ robust optimization results overlaid on deterministic level sets.

The solutions to the robust optimizations with $\sigma=0.03$ and $\sigma=0.04$ are substantially different from the other optimization results as shown in Fig 4(c), where they are also overlaid on the deterministic results. For these problems, the formerly inactive constraint on compliance became active. $A$ red (dark) solid region indicates where it is violated and wide red (dark) curves indicate where it is not violated. For two design variables and two active constraints, the solution must be at the intersection of the (robust) constraints, which moves the design points much farther from the deterministic solution.

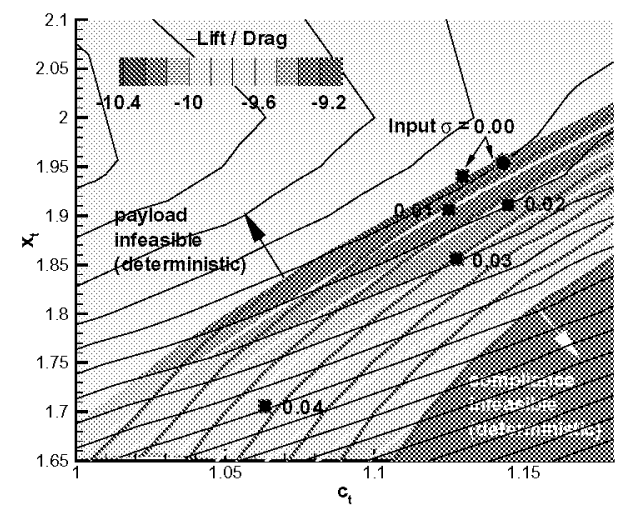

Figure 4(c). Probabilistic $\sigma=0.03 \& 0.04$ robust optimization results overlaid on deterministic level sets.

The effect on the planform shape can readily be seen in Fig. 5. Although the $\sigma=0.01$ and $\sigma=0.02$ cases are easily distinguishable from the deterministic case, they are indistinguishable from each other. The $\sigma=0.03$ and $\sigma=0.04$ cases are easily distinguishable from all other cases. Physically, the effect of reducing the 
setback is to decrease the twist deformation of the tip (wash-out), thereby increasing the lift and hence the available payload. But the increased lift, particularly at the tip, increases the bending and, with it, the compliance. The effect of increasing the tip chord is to increase the weight, but that effect is offset by the increased lift, which increases the available payload. This 2DV problem is not a representative engineering problem, but it is useful for understanding the robust design process through visualization and also for determining that the process is functioning properly. Moreover, the size of the coefficients of variation $\sigma_{i}$ used here is much larger than one would expect in practice for these selected variables.

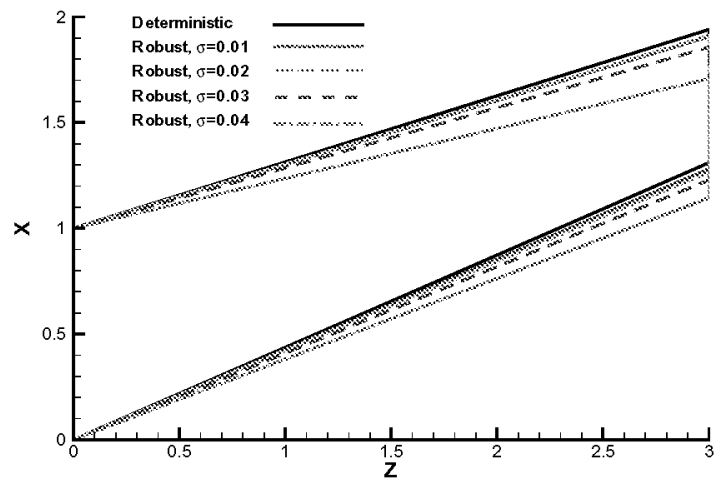

Figure 5. Planforms resulting from 2DV optimizations.

\section{Four-Design-Variable Cases}

Although the 2DV problem is useful for understanding the process and ascertaining that it is performing correctly, the variance of the design variables was necessarily exaggerated to see any effect. That is, the first step of the robust design process, quantification of uncertainty of the input variables, was not followed. For this 4DV example, a sensitivity analysis was performed to determine those parameters for which a realistic variance would have a significant effect on the output functions used as the objective and constraints. The four parameters chosen as design variables were the root airfoil section maximum thickness $t_{r}$, the root airfoil section maximum camber $\mathrm{Z}_{\mathrm{r}}$, and the structural sizing factors for the two inboard regions, $\Gamma_{1}$ and $\Gamma_{2}$, as shown in Fig. 1. For the cases shown here, a coefficient of variation, $\sigma_{i}=0.001$, was chosen for all four variables. As in the $2 \mathrm{DV}$ cases, a deterministic optimization was performed first. That deterministic result was used as the initial design for all the robust optimizations. The deterministic optimization process reduced the section thickness $t_{r}$ to reduce the shock strength thereby reducing the drag and improving the L/D. As a consequence, the wing became more flexible. To satisfy the compliance constraint $g(V)$, the structure element thickness increased, and, as a consequence, the wing became heavier. To satisfy the payload constraint $g(L-W)$, the section camber increased. The pitching moment did not appear to be affected sufficiently for that constraint, $\mathrm{g}\left(\mathrm{C}_{\mathrm{m}}\right)$, to be active. The deterministic results are shown in the first column of Table 1.

Table 1 and Figs. 6-8 present the results for the deterministic optimization and robust optimizations for several values of $\mathrm{k}$. An increase of the parameter $\mathrm{k}$ represents an increase in the specified target probability and therefore the probability that the constraints are met. Assuming a normal Gaussian distribution of the output variables, values of $\mathrm{k}=1,2$ and 3 would represent probabilities of $84.13 \%$, $97.73 \%$ and $99.87 \%$, respectively. The mean values of the constraint functions are shown in comparison to the robust constraint values in Table 1 . The design variables are compared in Fig. 6.

Table 1. 4DV problem results.

\begin{tabular}{|c|c|c|c|c|c|c|c|}
\hline & $\begin{array}{l}\text { Deterministic } \\
\text { solution }\end{array}$ & \multicolumn{2}{|c|}{$\begin{array}{l}\text { Robust solution, } \\
\qquad \mathrm{k}=1\end{array}$} & \multicolumn{2}{|c|}{$\begin{array}{l}\text { Robust solution, } \\
\mathrm{k}=2\end{array}$} & \multicolumn{2}{|c|}{$\begin{array}{c}\text { Robust solution, } \\
k=3\end{array}$} \\
\hline \multicolumn{8}{|c|}{ Design variable inputs } \\
\hline$t_{r}$ & 0.778 & \multicolumn{2}{|c|}{0.774} & \multicolumn{2}{|c|}{0.774} & \multicolumn{2}{|c|}{0.773} \\
\hline$z_{r}$ & 1.138 & \multicolumn{2}{|c|}{1.139} & \multicolumn{2}{|c|}{1.139} & \multicolumn{2}{|c|}{1.14} \\
\hline$\Gamma_{1}$ & 4.075 & \multicolumn{2}{|c|}{4.05} & \multicolumn{2}{|c|}{4.009} & \multicolumn{2}{|c|}{3.984} \\
\hline$\Gamma_{2}$ & 3.7 & \multicolumn{2}{|c|}{3.656} & \multicolumn{2}{|c|}{3.563} & \multicolumn{2}{|c|}{3.528} \\
\hline Responses & & robust & mean & robust & mean & robust & mean \\
\hline obj & -19.1 & -19 & & -18.95 & & -18.93 & \\
\hline$g(L-W)$ & -0.00077 & 0.000048 & -0.0107 & 0.00031 & -0.0211 & -0.000745 & -0.0328 \\
\hline$g(V)$ & -0.00032 & -0.000028 & -0.0077 & -0.00501 & -0.0203 & 0.000449 & -0.0225 \\
\hline$g\left(C_{m}\right)$ & -0.0666 & -0.0531 & -0.062 & -0.0422 & -0.0601 & -0.0262 & -0.0532 \\
\hline $\begin{array}{l}\text { L/D } \\
\text { weight }\end{array}$ & $\begin{array}{r}21.85 \\
54243\end{array}$ & & $\begin{array}{r}21.82 \\
53917\end{array}$ & & $\begin{array}{r}21.77 \\
53056\end{array}$ & & $\begin{array}{r}21.75 \\
52684\end{array}$ \\
\hline
\end{tabular}

